Journal of Ancient Philosophy ISSN 1981-9471 - FFLCH/USP www.revistas.usp.br/filosofiaantiga
J. anc. philos. (Engl. ed.), São Paulo, v.13, n.2. p. 35-64, 2019.

DOI: http://dx.doi.org/10.11606/issn.1981-9471.v13i2p35-64

\title{
Asclepio: Ecco il tuo Politico! \\ La relazione etico terapeutica fra medicina e politica in Platone
}

\author{
Barbara Botter
}

The purpose of this paper is to examine the political dimension of the relationship between the doctor and the patient, and the therapeutic dimension of the relationship between the politician and the citizen in Plato's philosophy. The purpose of the study is to show that the liaison between medicine and politics in the Platonic doctrine is not extrinsic, as if it were two disciplines analogous but of a different nature. We believe, on the contrary, that the relationship between the two sciences is substantial, by virtue of the political and therapeutic dimensions that both sciences essentially share. This affinity is possible as a result of the particular constitution of their object of study; the epistemological structure of the two sciences and the specific use of the persuasive discourse that both arts adopt to achieve their results. In order to examine our hypothesis we will investigate some passages from the Politicus, the Timaeus and the Laws.

\section{Introduzione}

L'approssimazione di medicina e politica non è nuova né sorprendente ed è riportata dall'Antichità ai nostri giorni. È evidente, infatti, che la malattia influenza il destino di un individuo, condiziona la vita sociale e l'economia di una città (Van der Ejik 2009, 11-12). Inversamente, cambiamenti nella struttura sociale e politica hanno ricadute a livello della salute individuale e collettiva. Grmek (1983, 141-2, apud Matsui 2015, 7576), nota che la trasformazione sociale nell'Atene del VI secolo provocò un declino nella salute e nella prospettiva di vita dei cittadini dell'epoca. La città greca passò da un regime agricolo ad una economia di commercio a seguito di una massiccia urbanizzazione, la quale provocò "o abandono da drenagem dos pântanos, a superpopulação urbana e a insuficiência de esgotos e inspeção sanitária. Além disso, o deslocamento dos refugiados da guerra trouxe doenças gastrointestinais, malária, tifoide e tuberculose" (Matsui 2015, $75)$. 
Journal of Ancient Philosophy ISSN 1981-9471 - FFLCH/USP www.revistas.usp.br/filosofiaantiga
J. anc. philos. (Engl. ed.), São Paulo, v.13, n.2. p. 35-64, 2019.

DOI: http://dx.doi.org/10.11606/issn.1981-9471.v13i2p35-64

Nei dialoghi di Platone il paragone fra politica e medicina è adoperato in modi eterogenei e a partire da variegati punti di vista ${ }^{1}$, cosicché risulta difficile trovare un filo conduttore che giustifichi e renda uniformi tutti i passi in cui Platone ricorre ad essa. Riferendosi al parallelo fra medicina e politica con ragguardevole frequenza, Platone si conforma ad un uso consolidato. Tucidide si era ispirato all'arte medica per inaugurare una interpretazione del passato da cui nacque la storia. ${ }^{2}$ Alcmeone usava termini di conio politico come, per esempio, monarchia e isonomia, per indicare lo stato degli umori corporei. Il legame fra medico e capo della città appare ancora in Pindaro ed Eschilo. ${ }^{3} \mathrm{Ma}$ l'interrelazione della medicina con la politica è, soprattutto, il punto di partenza della filosofia politica di Platone, dal momento che l'analisi del filosofo parte dall'osservazione della situazione malata di Atene.

Nei libri IV e IX delle Leggi Platone sfrutta l'analogia fra medico e malato come un paradigma per chiarire la relazione fra governante e governato e differenziare il governo giusto dal governo tirannico (Leges IV 720; IX 857) ${ }^{4}$, mentre nel libro X Platone, presentando gli dei come "supervisori dell'intero mondo", confronta la loro forma di governo con l'attività medica (Lombard 2016, 13). Nel Fedro il filosofo attribuisce un ruolo centrale all'uso che la medicina fa del logos al fine di evidenziare l'importanza della retorica filosofica (Phaedrus 211a sg.; cf. Gorg. 514a sg.). In particolare, i procedimenti dialettici della retorica sono giustapposti ai pharmaka ${ }^{5}$ usati nella medicina. ${ }^{6}$ Nel Gorgia la medicina è utilizzata come modello sul quale testare la forza razionale delle scienze in contrasto con la pratica empirica (Gorg. 464b; 500e-501a; 521e). Nella Repubblica e nel Timeo l'approssimazione della medicina alla politica è giustificata dal rapporto di specularità che vige fra i rispettivi oggetti di studio (Rep. 5405 d sg.; 408e; Tim. 44d sg.;

\footnotetext{
${ }^{1}$ La relazione fra política e medicina ampiamente documentata in Schul 1960 e Vegetti 1995.

${ }^{2}$ Tucidide, La Guerra del Peloponneso, VI 14.

${ }^{3}$ Si veda su questo punto Jouanna 1980, 299-319. In particolare 301-2; Lombard 2016 e Matsui 2015.

${ }^{4}$ Per citare i dialoghi platonici useremo le seguenti abbreviazioni: Tim. Timeo; Rep. Repubblica; Leges Leggi; Prot. Protagora; Phileb. Filebo; Phaedrus. Fedro; Phaedo Fedone; Stat. Politico; Gorg. Gorgia; Crat. Cratilo; Charm. Carmide, Ippia Min. Ippia Minore; Ion. Ione; Lis. Liside.

${ }^{5}$ Nella traslitterrazione del testo greco ci serviamo delle norme di traslitterazione fornite dalla rivista Archai. Novas Normas de transliteração. Archai n. 12, 193-194.

${ }^{6}$ Lo stesso riferimento al logos della medicina si trova nelle Leggi IV 720a6-c3; IX 857c4-e1.
} 
Journal of Ancient Philosophy ISSN 1981-9471 - FFLCH/USP www.revistas.usp.br/filosofiaantiga
J. anc. philos. (Engl. ed.), São Paulo, v.13, n.2. p. 35-64, 2019.

DOI: http://dx.doi.org/10.11606/issn.1981-9471.v13i2p35-64

69d sg. 73c-d; 75b-c; 87e sg.; 90a sg.;). L'esaltazione della medicina ricorre ancora nel Carmide, nel Filebo, nel Fedone, nell'Ippia Minore, nello Ione e nel Liside (Charm. 156d sg. Phileb. 55d sg.; Phaedo 96b sg.; Ippia Min. 375b; Ion. 538b-c; Lis. 209d-210c). Non mancano, infine, testi in cui la medicina è criticata, come è il caso dei primi libri della Repubblica, ma anche in questa occasione la polemica platonica è inserita in un contesto politico. $^{7}$ Sebbene già molti scritti siano stati dedicati agli aspetti della medicina nel Corpus Platonicum, la maggior parte di essi si concentra sulla funzione metodologica di essa a partire dalle pagine del Fedro dedicate da Platone a Ippocrate (Phaedr. 270). ${ }^{8}$

Nel presente testo affronteremo il legame fra medicina e arte del governo, evidenziando il fatto che entrambe le scienze si prendono cura dell'uomo nella totalità delle sue dimensioni fisica, psichica e socio politica, e utilizzano essenzialmente lo stesso strumento: la forza del logos. In vista di ciò esamineremo, principalmente, alcuni passi tratti dal Politico, dal Timeo, e dalle Leggi, servendoci di passi tratti da altri dialoghi quando se ne manifesti la necessità.

Dopo una breve presentazione delle due figure in questione $(\S 1)$, descriveremo $i$ modi in cui i medici e i politici si prendono cura rispettivamente dei pazienti e dei cittadini (§2); evidenzieremo, quindi, quali attributi distinguono le immagini dei due professionisti dalle loro imitazioni (§3); per chiudere con l'analisi del tipo di discorso che essi utilizzano per attingere il loro obiettivo ( $\$ 4$ e 5).

\section{§ 1) Chi cura l'uomo: il medico o il governante?}

Il legame fra governante e medico, da un lato, e cittadino e paziente, dall'altro, diventa più significativa qualora si tengano a mente due indicazioni: 1. che qualsiasi techne è definita dal suo oggetto; 2. quale sia lo scopo della politica per Platone.

\footnotetext{
${ }^{7}$ Su questo punto si veda Matsui 2015.

${ }^{8}$ Sulla funzione della medicina come modello metodologico di indagine, si veda Marino 2018; Lombard 2016. Nel Fedro Socrate e Fedro conversano all'ombra di un platano presso le rive rive del fiume Illisso sul tropos, ossia sulla maniera di procedere dell'arte medica e lo relazionano con le procedure dell'arte oratoria, evidenziando i fondamenti razionali dell'arte medica (Phaedrus $270 \mathrm{~b}-\mathrm{e}$ ). L'intera conversazione su questo tema è governata dal metodo (methodou) in uso da parte di Ippocrate per comprendere la realtà naturale (peri physeos) (Phaedrus 270d).
} 
Journal of Ancient Philosophy ISSN 1981-9471 - FFLCH/USP www.revistas.usp.br/filosofiaantiga
J. anc. philos. (Engl. ed.), São Paulo, v.13, n.2. p. 35-64, 2019.

DOI: http://dx.doi.org/10.11606/issn.1981-9471.v13i2p35-64

1. Nell'Alcibiade 128a-129a Platone riflette sul fatto che il tecnico deve conoscere la natura dell'oggetto e il modo per prendersene cura se vuole padroneggiare la propria arte. Nello Ione ritorna sull'idea che la specificità di ogni tecnica è determinata dalla specificità del suo oggetto: "Ciò che si conosce attraverso la medicina, tale è l'oggetto che, in ultima analisi, definisce la medicina" (537d; cf. Lis. 209d-210c). Ora, tanto la medicina quanto l'arte politica si prendono cura dell'uomo. Gli attributi che discriminano le due arti emergeranno nel seguito del testo. 2. Vegetti (2010, 32-41) evidenzia che quando il filosofo si serve del termine ta politika pensa a) al fatto che, essendo la società in una condizione di malattia ${ }^{9}$, è necessario un medico della città che possa curarla; b) alle strategie terapeutiche e educative che propiziano il ristabilimento della salute, la cui efficacia depende dalla corretta distribuzione degli incarichi politici e dalla struttura economica e sociale della città.

La prossimità fra le figure del medico e del governante viene sottolineata da Platone all'esordio del Politico.

In questo dialogo la preoccupazione di Platone è di definire la figura del politico e di distinguerla dalle sue imitazioni. Ciò significa che all'epoca del filosofo il politico ancora non aveva attinto dei contorni distinti e veniva facilmente confuso con le immagini che aspiravano ad assumerne le veci. Inoltre, prima di Platone, non c'è distinzione fra scienza (episteme) e techne, dal momento che entrambe i termini indicavano una certa competenza e solo con Platone e Senofonte l'insegnamento teorico viene distinto dall'insegnamento pratico (Lombard 2016, 24). ${ }^{10}$ Tuttavia, questa distinzione non si trova compiuta già negli scritti platonici: arti, tecniche e conoscenza empirica non hanno trovato ancora delle delimitazioni in grado di definirli.

E se il politico, negli scritti platonici, deve lottare per mostrare la propria esistenza, autonoma e distinta da quella del retore e del sofista, la stessa incertezza pesa sulla classe dei medici.

\footnotetext{
${ }^{9}$ Dirà Platone nella Repubblica 372a-373d (cf. 374a; 407a-410a) che la società è travagliata dall'infiammazione per aver abusato delle leccornie e delle prelibatezze della culinaria siciliana. E nel Gorgia l'anima umana è presentata come "cancrenosa sotto la cicatrice", quindi incurabile (519c-520a). Si veda LOMBARD 2016, 16 e nota 35.

${ }^{10}$ Per una più ampia riflessione sulla distinzione fra episteme e techne si veda Lombard 2016, in particolare 29-57.
} 
Journal of Ancient Philosophy ISSN 1981-9471 - FFLCH/USP www.revistas.usp.br/filosofiaantiga
J. anc. philos. (Engl. ed.), São Paulo, v.13, n.2. p. 35-64, 2019.

DOI: http://dx.doi.org/10.11606/issn.1981-9471.v13i2p35-64

"Techne superiore, scienza della salute, filosofia del corpo, filosofia naturale, filosofia del tutto, nuovo settore della cultura e della vita intellettuale, la medicina è, nel IV secolo, tanto diversa quanto prestigiosa. Non si offre come una disciplina distinta, autonoma e stabilita" (Lombard 2016, 24-25).

Come spiega Desclos (2013), estiste una divergenza all'epoca di Platone relativa a chi goda a pieno diritto del titolo di medico (cf. Laks 2013, in particolare 67). I medici sono confusi con i sofisti, con i poeti, con gli indovini e con i filosofi e Platone non si dirime dall'alterco. ${ }^{11}$ Nel libro I della Repubblica, nel corso della discussione con Trasimaco, i due interlocutori si richiamano alla definizione del "vero" medico, lasciando intendere che il termine da solo non godeva ancora di sufficiente chiarezza $(R$. 340d341a). Una pallida definizione della medicina è fornita da Platone nel Gorgia, quando Socrate la cita in quanto arte che libera gli uomini dalla malattia, come l'arte di procurare la ricchezza libera dalla povertà e la giustizia libera dalla malvagità (Gorg. 478a-d).

I medici di formazione ippocratica sentono la necessità di definire una precisa epistemologia per distinguere la loro arte dalle pratiche dei sacerdoti e dei filosofi presocratici. Frutto di tali sforzi è il processo di progressiva desacralizzazione e laicizzazione delle conoscenze mediche (Lopez 2004, 16) messa in atto, in particolare nei trattati Sulla Medicina e Sull'Arte. ${ }^{12}$ "Neste esforço de definição epistemológica, os outros autores hipocráticos definem o que é a medicina em relação ao campo de objetos dos quais se ocupa e ao método que ela aplica na pesquisa e na prática" (Marino 2018, 351). Prende piede, in questa maniera, quella che Bourgey definisce "médicine rationnelle" (1953, 191), visto che alla raccolta dei dati per via di esperienza si accompagna costantemente “un égal souci de la raison” (Bourgey 1953, 193).

Analogamente sul versante politico, lo Straniero, nel Politico, si preoccupa di distinguere la figura del politico da altre figure che con lui potrebbero rivaleggiare e fra gli emuli appare la figura del medico, accanto ai commercianti, agli agricoltori, ai fornai e ai maestri di ginnastica (Stat. 268a). Il medico assomiglia al politico, nella misura in cui entrambe realizzano una professione direttiva (epitaktike, Stat. 259d6-260c5) e formativa, nel senso di generare un certo tipo di uomo. Ciò che distingue i medici dai politici è il

${ }^{11} \mathrm{Si}$ vedano al proposito le riflessioni contenute nei trattati ippocratici sulla necessità di distinguere i medici dai filosofi e dai sacerdoti, in particolare nel trattato Medicina Antica XX. Per approfondimenti si veda Mesquita 1998, 63-85.

${ }^{12}$ Ampie riflessioni sui due trattati medici citati si trovano in Cambiano 1991. 
Journal of Ancient Philosophy ISSN 1981-9471 - FFLCH/USP www.revistas.usp.br/filosofiaantiga
J. anc. philos. (Engl. ed.), São Paulo, v.13, n.2. p. 35-64, 2019.

DOI: http://dx.doi.org/10.11606/issn.1981-9471.v13i2p35-64

fatto che la professione del medico è sostanzialmente pratica e i medici si occupano di un paziente per volta. L'arte politica, invece, è un tipo di professione essenzialmente direttiva, ossia, il politico non agisce direttamente ma fa agire gli altri; inoltre, egli si prende cura dell'uomo nella totalità dei membri della società. ${ }^{13}$

Dopo aver concluso la narrazione del mito di Crono, lo Straniero attualizza la dieresi che deve concludere alla definizione del politico. In questa ripresa, alcuni dei termini anteriormente utilizzati vengono di proposito sostituiti. Nelle tappe della dieresi anteriori al mito di Crono, lo Straniero riconosce di aver attribuito al politico la capacità di allevare (throphein) il corpo sociale (Stat. 275b1-4), in modo simile a quanto fa un pastore in relazione al proprio gregge e al pari di Crono in rapporto al genere umano. Nella ripresa della definizione, il termine trophe viene invece sostituito col termine therapein. "Os pastores mereceriam de facto o nome de tropheus (e o deus parcialmente [...]), mas não o rei” (Loureiro 2011, 125). Il genere a cui anche l'attività del politico appartiene, e di cui il nutrire è una specie, è la therapeia (Stat. 275e3), ossia una prestazione di servizio che ha come fine lo stato di salute collettiva.

"Tutti i pastori hanno in comune con gli altri l'attività di allevare (trephein) ciascuno il proprio gregge, ma l'uomo politico no: eppure gli abbiamo attribuito questo nome, mentre dovevamo attribuirgliene uno diverso, che valesse in comune per tutti. [...] E come potrebbe non essere comune in qualche modo a tutti il termine "aver cura" (therapeuein, 275e3), senza che sia specificato come allevamento (trophes) o come qualche altra determinata attività (meden tinos alles pragmateias)? Anzi, se l'avessimo chiamata arte di assistere il gregge (agelaiokomiken), o di accudirlo (therapeutiken), o comunque di prendersene cura in generale (epimeletiken), avremmo potuto includere anche l'uomo politico insieme con gli altri, dal momento che il nostro discorso aveva il compito di determinare esattamente questo significato" (Stat. 275d8-276a. Traduzione di C. Mazzarelli).

Lo Straniero fa notare che i termini più appropriati per la definizione di politico sono komide, therapeia, epimeleia, che traduciamo rispettivamente per assistenza, capacità di accudire, e cura, e fra i tre termini, therapeutiken è il termine più coerente, visto che indica la cura (epimeleia) rivolta all'intera comunità umana e arte del governo di tutti gli individui (Loureiro 2011, 86).

Evidenzia con precisione questo punto Gerbier:

13 "O político não é médico dos humanos, mas organiza um serviço nacional de saúde" (Loureiro 2011, 87). 
Journal of Ancient Philosophy ISSN 1981-9471 - FFLCH/USP www.revistas.usp.br/filosofiaantiga
J. anc. philos. (Engl. ed.), São Paulo, v.13, n.2. p. 35-64, 2019.

DOI: http://dx.doi.org/10.11606/issn.1981-9471.v13i2p35-64

La formule du nouvel espace lexical dans lequel on va rechercher l'homme politique pour le distinguer des autres offices de la cité est donc le suivante: le terme générique qui aurait été preférable est au croisement de l'agelaiokomike techne (art du soin des tropeaux) qui remplace agelaiotrophike (art du nourrissage des tropeaux), usité en 261e; de la therapeutike techne que la phrase précédente a semblé définir comme paradigmatique (ou plus paradigmatique que les autres); et de l'epimeletike techne qui reprend le même terme constant, epimeleia, dominant tout le passage" (Gerbier 2003, 4).

La definizione dialettica formulata nella parte anteriore al mito di Crono non viene semplicemente abbandonata, ma richiede di essere appurata essendosi rivelata generale e, perciò, astratta. L'assenza di Crono nella direzione della società lascia spazio alla nascita della politica e della medicina. Infatti, sotto il governo della divinità né l'una né l'altra ricoprivano un ruolo essenziale, visto che il dio sopperiva a tutti i bisogni e la vita umana non era soggetta alle malattie. La situazione idillica svanisce al ritrarsi di Crono: politica e medicina sono i sintomi del disequilibrio sociale creatosi con il ritrarsi di dio. ${ }^{14}$

Nel seguito vedremo più dettagliatamente in cosa consiste l'attività di cura propria del politico, in che misura essa detiene una certa affinità con l'attività del medico e in che misura si distanzia. In particolare, affronteremo i seguenti aspetti della relazione fra le figure dei due professionisti: 1) entrambe attendono alla cura dei corpi e delle anime degli uomini, perché, alla luce degli scritti platonici a partire dai quali muoviamo le nostre riflessioni, non è giustificato ignorare l'una o l'altra delle due dimensioni umane; 2) ciò che distingue i veri medici e i politici autentici dalle loro imitazioni è il dominio della scienza e della conoscenza; 3 ) tanto nel caso della relazione fra medico e malato quanto nel legame fra governante e governato il rapporto fra il superiore e i subordinati è gestito attraverso un uso attento del logos.

\section{§ 2) La relazione "duale” fra anima e corpo}

\footnotetext{
${ }^{14}$ Analogamente, nella Repubblica la presenza della medicina è segno di una città malata. La medicina che cura le malattie sorte in seguito alla vita sedentaria e al regime non sano assunto dagli uomini prova che la città non è stata educata. In verità, questo tipo di medicina non cura gli uomini ma ne prolunga la sofferenza: uomini che sono morti in relazione ad una vita resa felice dalla pratica della virtù e dei valori sono curati da questa medicina esattamente nella loro patologia. Platone usa un termine significativo per designare questo tipo di cura: nosotrophia, ossia nutrizione della malattia (Rep. III 407b1). Cf. Casertano 2011, 52.
} 
Journal of Ancient Philosophy ISSN 1981-9471 - FFLCH/USP www.revistas.usp.br/filosofiaantiga
J. anc. philos. (Engl. ed.), São Paulo, v.13, n.2. p. 35-64, 2019.

DOI: http://dx.doi.org/10.11606/issn.1981-9471.v13i2p35-64

Vediamo anzitutto le ragioni per cui il medico e il governante curano tanto il fisico quanto la dimensione psichica dell'uomo. Siamo persuasi, infatti, che, grazie al sostegno della dottrina ippocratica testimoniata nel Fedro e alla luce dell'antropologia del Timeo, non sia più possibile trattare corpo e anima separatamente. ${ }^{15}$

Nel Fedro Socrate attribuisce ad Ippocrate l'indagine sulla "natura del tutto

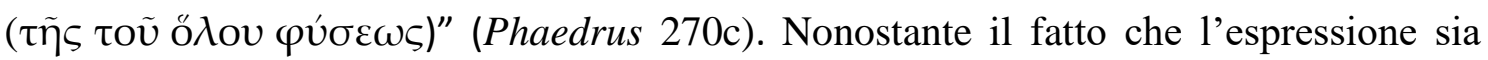
stata, fin dall'antichità, motivo di disputa fra gli esegeti relativamente al significato che si debba attribuire al termine holos $^{16}$, ossia totalità, sembra fuori discussione che l'oggetto proprio dell'arte medica sia non solo il corpo, ma il sinolo psico somatico e la relazione fra l'uomo e la natura (Lopez 2004, in particolare, vol. I, 39-50).

Nel Timeo Platone fa leva su un rapporto fra anima e corpo tale da non separare le due dimensioni al punto da rendere la loro relazione conflittuale; né le confonde in modo da creare una unità indistinguibile. Utilizzando un ricorso grammaticale in uso nel dialetto attico, riteniamo che il legame fra anima e corpo possa dirsi "duale". Come afferma Monique Dixsaut:

"L'âme n'est donc pas pour Platon une substance séparée du corps, et si elle doit s'en séparer, sa separation est la conquête d'une pénsée que le corps n'alourdit plus mais où elle est déjà présente. [...] Partout où il y a mouvement et vie il y a aussi âme et une sorte de pénsée et il y a jusque dans le corps, une sagesse" (Dixsaut 2015, rispettivamente 57 e 58).

Anima e corpo non sono divisi di tal modo da essere incompatibili, né fusi in una unità che impedirebbe qualsiasi relazione. Essi sono legati l'uno all'altro, questo "altro" non essendo, però, "uno tra gli altri” (Dixsaut 2015, 58).

La "ghiandola pineale" platonica, ossia gli elementi che rendono duale la relazione fra il fisico e lo psichico sono tre, ossia a) il fatto che l'anima sia definita da Platone, dal Fedro alle Leggi e, secondo aluni interpreti nel Timeo (Robinson 2018, 144-148; Carone 2008, in particolare capitolo III, 87-123), come un movimento; b) il fatto che tanto il

\footnotetext{
${ }^{15}$ Si veda al proposito gli studi di Dulce Reis 2010; Morais 2009a; Frias 2001; Stell 2001; Brisson 2015, i quali mettono in rilievo la differenza e la inseparabilità delle due componenti.

${ }^{16}$ I significati ricorrenti, secondo lo studio di Lopez, sono i seguenti: “a) natura o universo; b) ambiente; c) corpo; d) oggetto o problema in discussione; e) coapparteneza corpo-anima; f) "sistema di eventi" che comprende "tutta la natura" e "tutto il sapere" (Lopez 2004, 39-40).
} 
Journal of Ancient Philosophy ISSN 1981-9471 - FFLCH/USP www.revistas.usp.br/filosofiaantiga
J. anc. philos. (Engl. ed.), São Paulo, v.13, n.2. p. 35-64, 2019.

DOI: http://dx.doi.org/10.11606/issn.1981-9471.v13i2p35-64

movimento psichico quanto il movimento fisico sono matematicamente costituiti; c) il fatto che l'anima è "essenzialmente" in relazione con il corpo.

Vediamo in dettaglio.

L'anima è movimento: "Qual è la definizione di ciò il cui nome è "anima"? Ne abbiamo un'altra oltre a quella detta or ora, ossia "quel movimento che è capace di muovere se stesso" (Leges X 896a). Se l'anima è essenzialmente movimento, non sorprende che essa sia turbata, positivamente o negativamente, dai movimenti provenienti dal corpo e da movimenti prodotti nei fenomeni esterni, come è il caso della musica o delle pratiche mediche (Ferrari 2016, 99 e 131).

Il ruolo del movimento interno o esterno al corpo, nel Timeo, è a tal punto rilevante da permettere a Timeo di affermare che è compito del medico classificare i tipi di movimento che gli permettano di armonizzare anima e corpo affinché la salute non venga turbata o venga riequilibrata, qualora perduta. ${ }^{17}$ Il filosofo, nella Repubblica, attribuisce un particolare ritmo musicale alla meschinità (aneleutheria), alla violenza (hybris) e alla pazzia (mania) e ipotizza, ispirato dalla teoria musicale di Damone, che se la pazzia ha un movimento musicale che la suscita e ne accresce l'intensità, deve esistere anche un ritmo che la placa e che la cura.

"Potremmo anche unirci a Damone per decidere quali sono i ritmi che traducono la volgarità, la violenza, oppure la pazzia e ogni altro difetto e quali invece vadano tenuti in serbo per ler qualità opposte" (Rep. 400b. Traduzione di G. Reale).

Trattasi di un'idea simile a quella che si ritroverà nelle Leggi, in cui, descrivendo gli elementi fondamentali per uno sviluppo sano dei bambini, lo Straniero di Atene cita il movimento. Ai bambini deve essere consentito di godere della condizione simile a quella vissuta dalle persone che si trovano in una nave. Per questo, le nutrici dondolano i neonati tra le braccia affinché vengano colti dal sonno. Con questa stessa tecnica, conclude Platone, si curano quelli che sono "come baccanti per la pazzia"

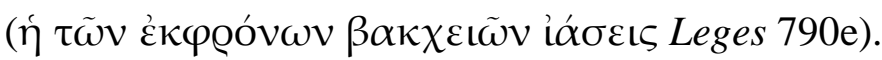

${ }^{17}$ Cf. Tim. 89a5-9: "La purificazione che si ottiene attraverso i movimenti di oscillazione sulle navi o su qualunque altro mezzo di trasporto che non susciti stanchezza". 
Journal of Ancient Philosophy ISSN 1981-9471 - FFLCH/USP www.revistas.usp.br/filosofiaantiga
J. anc. philos. (Engl. ed.), São Paulo, v.13, n.2. p. 35-64, 2019.

DOI: http://dx.doi.org/10.11606/issn.1981-9471.v13i2p35-64

L'azione del movimento indotto dall'esterno genera uno scuotimento maggiore a quello presente nell'animo e permette la restaurazione della calma e della moderazione (Leges 790d-791b). Illustra bene il pensiero platonico il commento di Casertano:

"Un movimento disarticolato, disordinato, scomposto sia del nostro corpo, ma principalmente della nostra anima, che viene chetato da un altro movimento che giunge dall'esterno a toccare l'intimo del nostro animo, e lo tranquillizza, lo calma, ne neutralizza i movimento inconsulti secondandoli e forzandoli e guidandoli all'ordine e alla compostezza, all'armonia: questa è la strategia terapeutica che la musica è in grado di attuare per farci diventare, da ekphrones, emphrones" (Casertano 1991, 50).

Avendo stabilito il movimento come denominatore comune fra anima e corpo possiamo dedurre che non esiste un movimento fisico che non sia allo stesso tempo psichico e una dynamis dell'anima che non aumenti o faccia defluire qualche umore corporeo, rinunciando a qualsiasi tipo di dualismo.

Questo primo punto è legato al successivo (punto b) senza soluzione di continuità. I movimenti fisico e animico hanno una struttura matematica realizzata sulla base di precise proporzioni numeriche (Leges X 896e-897a). ${ }^{18}$

"Le serie di movimenti hanno, nel loro accadere, una struttura matematico-armonica, infatti la trasmissione di moto attraverso la vibrazione può darsi con velocità diverse. La differente configurazione matematica di questi movimenti [...] rende possibile la comunicazione di anima e corpo. Entrambi questi enti hanno infatti un'intelaiatura matematica, e dunque la matematica può fungere da interfaccia fra di loro" (Ferrari 2016, 105). ${ }^{19}$

Tale quale il cosmo, l'uomo è costituito, al livello fisico, degli stessi triangoli elementari che compongono tutti gli enti e che rappresentano, in combinazione matematica, gli elementi di cui tutti i membri sono costituiti (Brisson 2015, 420-429, in particolare 423), sebbene nell'uomo gli elementi cosmici non siano presenti allo stato puro e nella pienezza della loro potenza.

"Per quanto concerne la natura dei corpi di tutti gli esseri viventi noi osserviamo, in certo modo, che entrano nella loro costituzione fuoco, acqua, aria e terra. [...] che ciascuno di questi elementi è presente in noi solamente in piccola quantità e di poco conto e in nessuna parte e in nessun

${ }^{18} \mathrm{Si}$ veda al proposito Brisson 2001, 285-286; 2003, 189-207; 2015, 314-323; Fronterotta 2015, 202-203.

${ }^{19} \mathrm{Si}$ veda al proposito Dixsaut 2002, 61. 
Journal of Ancient Philosophy ISSN 1981-9471 - FFLCH/USP www.revistas.usp.br/filosofiaantiga
J. anc. philos. (Engl. ed.), São Paulo, v.13, n.2. p. 35-64, 2019.

DOI: http://dx.doi.org/10.11606/issn.1981-9471.v13i2p35-64

modo puro né dotato di una potenza degna della sua natura" (Phil. 29a. Traduzione di Claudio Mazzarelli).

Tanto il cosmo quando l'uomo sono strutturati in modo matematicamente proporzionato da una iniziale porzione di fuoco e terra a cui il Demiurgo ha aggiunto acqua e aria nella seguente proporzione: fuoco $:$ aria $=$ aria $:$ acqua $=$ acqua $:$ terra in rispetto alla legge di formazione dei solidi (Migliori, 2003, 101). Ciò che discrimina l'uomo rispetto all'universo è che, differentemente dall'universo, nell'uomo il legame fra i triangoli non comporta la stessa stabilità che nel cosmo (Tim. 32c1-4; cf. 32d1-33a e 33a6-b1; 37e-38a). ${ }^{20}$ Infatti, le radici dei triangoli che lo compongono sono fissate solo con chiodi (desmois) invisibili per la loro piccolezza (Tim. 43a2-3). Perciò, il movimento fisico nell'uomo, in particolare i cicli della respirazione e della nutrizione (Tim. rispettivamente $79 \mathrm{~b}$ e $80 \mathrm{~d}$ ), è destinato ad interrompersi.

Anche la dimensione animica è formata di proporzioni matematiche precisamente pensate dal filosofo per risultare in una complessa combinazione che può essere ridotta alla ripetizione di tre frazioni: 3/2, 4/3, 9/8. ${ }^{21}$ Queste frazioni sono particolarmente significative, visto che corrispondono rispettivamente agli intervalli musicali di quinta, di quarta e di ottava della scala diatonica (Del Forno 2005, 22), evidenziando, perciò, il movimento "musicale" dell'anima.

Infine, il fatto che l'anima sia fin dall'origine in rapporto con il corpo (punto c), traspare dall'analisi attenta delle pagine dedicate alla costituzione dell'anima immortale dal Timeo. Nell'omonimo dialogo, il protagonista descrive la composizione dell'anima cosmica, di cui l'anima umana è un derivato. L'anima cosmica è costituita di due cerchi, a loro volta formati dalla mescolanza di tre ingredienti intermedi rispettivamente Essere Indivisibile e Essere Divisibile, Identico Indivisibile e Divisibile, Diverso Indivisibile e Divisibile. Visto che i termini Divisibile e Indivisibile rappresentano rispettivamente la sfera sensibile e quella intelligibile (Brisson 2015, 269-275; Robinson 2018, 111-112) è

\footnotetext{
${ }^{20}$ Visto che, nel suo insieme, il cosmo non è circondato da nulla al proprio esterno, l'universo non è soggetto alla dissoluzione e alla distruzione. Si veda Brisson 2001, in particolare 273, e nota 714 .

${ }^{21}$ Sull'elaborazione dei calcoli matematici che Platone realizzò si vedano gli eccellenti lavori di Brisson 2001, 285-286; 2003, 189-207; 2015, 314-323; Del Forno 2005, 22-23, e Ferrari 2016, 108-113.
} 
Journal of Ancient Philosophy ISSN 1981-9471 - FFLCH/USP www.revistas.usp.br/filosofiaantiga
J. anc. philos. (Engl. ed.), São Paulo, v.13, n.2. p. 35-64, 2019.

DOI: http://dx.doi.org/10.11606/issn.1981-9471.v13i2p35-64

evidente che le due dimensioni sono fin dall'origine poste in relazione giustamente per il fatto che l'anima stessa nella sua essenza rappresenta un metaxy (Ferrari 2016, 156).

Da quanto esposto si può trarre la seguente conclusione: se l'anima è essenzialmente in relazione con il corpo, in quanto è il metaxy che media i movimenti matematicamente articolati, qualsiasi alterazione in un assetto della struttura antropologica si ripercuote nell'altro e provoca una alterazione nella struttura psico fisica e nella condotta degli individui. Perciò, il medico e il governante potranno agire in una delle due dimensioni prevalentemente, o in entrambe simultaneamente e attraverso pratiche differenti, coscienti del fatto che l'alterazione si ripercuoterà sulla totalità dell'individuo.

Il medico agisce sull'unità físico psichica attraveros la diaita ${ }^{22}$, ossia influenza il corpo attraverso l'alimentazione e le attività fisiche e interviene sull'anima attraverso il discorso persuasivo. ${ }^{23}$

Il politico si prende cura dei cittadini attraverso una paideia che fa leva sulle due componenti tradizionali della ginnastica per agire sul corpo e della musica come pratica psicagogica. $^{24}$

\section{§3) Governanti, medici, leggi e codici a confronto}

Il dovere e la legittimità di agire sulla totalità delle componenti umane in vista di ciò che stimano il bene dei subordinati, deriva al medico e al governante dal possesso della scienza e della conoscenza (episteme) delle rispettive arti. Infatti, è essenziale discriminare le tecniche che hanno una reale conoscenza dei rispettivi oggetti da quelle

\footnotetext{
${ }^{22}$ Il termine diaita, in greco, riveste un senso bem più ampio dell'attuale "dieta", indicando più propriamente uno stile di vita che include l'alimentazione, le attività fisiche e tutto ciò che dall'esterno, in qualche modo, influenza l'individuo (Capriglione 2000, 155-172; Garcia Gual 2000, 43-67; Thivel 2000, 25-37). Scrive Garcia Soler: "Everything that is consumed by a man, as well as the activities he does, has an influence on his body and the purpose of dietetic medicine is to prevent the condition which may suppose a risk, offering a diet based on a balancing relationship between food and exercise" (Soler 2004, 5).

${ }^{23}$ Platone fornisce una definizione di medico come colui che "suscita benessere e vigore offrendo

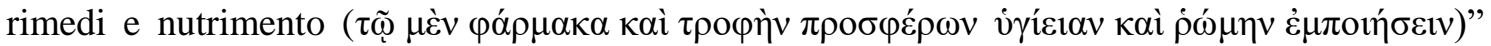
(Phaedrus 270b; cf. Ion 531d-432d). Gli effetti del discorso persuasivo del medico citati nei libri IV e IX delle Leggi saranno oggetto dell'item 5.

${ }^{24}$ Riprenderemo in dettaglio l'azione educativa del politico nell'item 5.
} 
Journal of Ancient Philosophy ISSN 1981-9471 - FFLCH/USP www.revistas.usp.br/filosofiaantiga
J. anc. philos. (Engl. ed.), São Paulo, v.13, n.2. p. 35-64, 2019.

DOI: http://dx.doi.org/10.11606/issn.1981-9471.v13i2p35-64

che, per quanto possano apparire attraenti, si riducono a procedure senza valore in quanto a conoscenza (Lombard 2016, 19).

Ciò che distingue il medico dalla sua imitazione, ossia dagli infermieri (hyperetai), non è l'esercizio della professione, bensì il possesso della scienza medica (Stat. 291d-303a). Allo stesso modo, ciò che distingue il buon governante dal tiranno è il possesso della scienza politica e non l'esercizio del potere sovrano. Su questo punto il Politico fa eco alle riflessioni della Repubblica. Il libro X delle Leggi chiarisce che agire seguendo una arte è sempre ricercare una certa perfezione. Per poter ricondurre il paziente allo stato di salute, il medico deve curare ciò che più importa, tralasciando le altre parti del corpo. Allo stesso modo, l'uomo di governo deve realizzare "esattezza e perfezione in ciò che è piccolo e in ciò che è grande" conformemente a quanto fanno gli intagliatori d pietre, perché "le pietre grandi senza le piccole non restano salde" (Leges 902d).

Chi domina la scienza politica è superiore anche alla legge, così come l'autorità del medico è superiore a qualsiasi trattato di medicina:

È quello che in misura non minore pensiamo dei medici: che ci curino con il nostro consenso, oppure senza, tagliando e bruciando o infliggendoci qualche altra sofferenza, seguendo regole scritte o non scritte, siano essi ricchi oppure poveri, nondimeno, in ogni caso, li chiamiamo medici, finché, facendo prescrizioni con arte (techne), purificandoci, sia con il togliere in vario modo qualcosa, sia anche con l'aggiungerlo, purché solo per il bene dei nostri corpi, rendendoli migliori da peggiori che erano, riescano, ciascuno con le sue cure, a salvare i corpi curati. È in questo modo che porremo, come credo, e non in un altro, che questa è la sola definizione corretta del potere medico e di qualsiasi altro potere" (Stat. 293b-c. Traduzione di C. Mazzarelli).

Il medico, al pari del politico, in quanto dotati della competenza che la conoscenza delle rispettive arti conferisce loro, sono esonerati dal rispettare gli obblighi imposti da trattati medici o dal corpo legislativo, dei quali, in ultima istanza, sono gli autori. ${ }^{25} \mathrm{E}$ la tecnica legislativa prodotta dal governante è superiore alle altre tecniche, anche alla tecnica giuridica (Gorg. 530b), dal momento che "procura evitar o aparecimento da maldade na alma, enquanto a técnica jurídica serve para tratar desta doença. A finalidade da legislação [...] é desviar do mal por meio da educação e alcançar a condição melhor

\footnotetext{
${ }^{25}$ Nel passo del Politico citato nel testo (293b-c), come nel Gorgia (4633a-465b), nell'Alcibiade (107c) e nello Ione (531e), la medicina è invocata per rafforzare l'idea della competenza tecnica. Scrive Soares, "[...] há uma edoné dietética aceitável, aquela que convive com a noção de béltiston, que no contexto médico [...] significa "melhor para a saúde". Que, em linguagem médica, falar do "melhor" corresponde a "saudável" e é o domínio do conhecimento do médico são ideias que ecoam nos diálogos" (2016, 241).
} 
Journal of Ancient Philosophy ISSN 1981-9471 - FFLCH/USP www.revistas.usp.br/filosofiaantiga
J. anc. philos. (Engl. ed.), São Paulo, v.13, n.2. p. 35-64, 2019.

DOI: http://dx.doi.org/10.11606/issn.1981-9471.v13i2p35-64

para o corpo e para a alma" (Lisi 2011, 206-207). Quando regna la competenza dello specialista, leggi, trattati o sanzioni passano in secondo piano, perché

[...] una legge non potrà mai ordinare con precisione la cosa più buona $\mathrm{o}$ più giusta per tutti, includendo insieme il massimo di equità. Infatti, le disuguaglianze degli uomini e delle azioni e il fatto che nessuna mai delle vicende umane porta, per cosí dire, tranquillità, non permettono neppure che alcuna arte - nessuna, quale che sia - possa dimostrare, in nessun campo, qualcosa di semplice e di valido per tutti i casi, e per tutto il corso del tempo. (Stat. 294b. Traduzione di C. Mazzarelli).

Alla scienza politica e alla scienza medica sono riconosciute tanto il rigore (akribeia), quanto quella capacità plastica che un congiunto di prescrizioni mediche e di norme politiche non possono vantare. Il rigore attribuito alla scienza medica è reso manifesto dalle parole di Trasimaco nel libro I della Repubblica. Il sofista insiste che, nel linguaggio rigoroso, il medico non può sbaglire e lo stesso vale per chi si proclama governante (Rep. 340d-e). ${ }^{26}$ Il medico può prescrivere al malato di seguire certe indicazioni nel momento in cui debba assentarsi, ma ha pieno diritto di ratificarle al suo ritorno. Come difende Gerbier (2003, 11 del testo on-line), la medicina non è una scienza di codici: i codici sono conditio sine qua non la medicina si costituisce come scienza, ma la scienza propriamente detta è incarnata nella figura del medico e non nella parola scritta.

Nel Fedone, in ossequio alla figura della seconda navigazione (99d), Platone allinea medicina e arte del governare per dimostrare quella che sarà la conclusione del Politico, ossia che non si deve sempre "lasciare a queste arti il diritto sovrano di comandare" perché un puro insieme di regole codificate ostacola la ricerca e impoverisce la conoscenza. La legge scritta è sempre un ripiego" (Stat. 297c-299b. cf. Lombard 2016, 20-21).

Il medico, in quanto detentore della scienza medica $(\kappa \alpha \tau \dot{\alpha}$

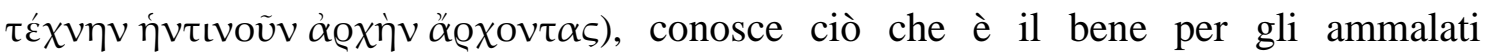

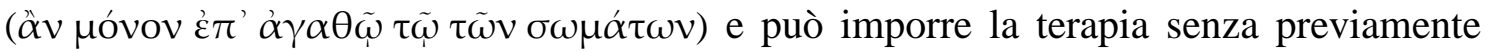

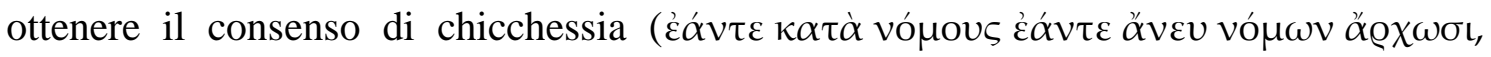

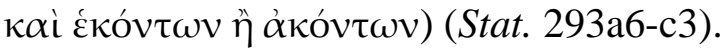

"Se uno senza persuadere colui che viene curato, ma possedendo correttamente l'arte, costringesse, contro le prescrizioni scritte, un ragazzo, un uomo, o anche una donna a seguire la

${ }^{26}$ Interessanti al proposito le riflessioni di Palumbo 2016, 38-39. 
Journal of Ancient Philosophy ISSN 1981-9471 - FFLCH/USP www.revistas.usp.br/filosofiaantiga
J. anc. philos. (Engl. ed.), São Paulo, v.13, n.2. p. 35-64, 2019.

DOI: http://dx.doi.org/10.11606/issn.1981-9471.v13i2p35-64

regola migliore, quale sarà il nome di questo atto di violenza? Non sarà forse qualunque nome tranne quello che si suole chiamare errore contro l'arte medica, cioè atto nocivo alla salute? E chi ha subito un tale atto di forza potrà dire, a ragione, tutto in proposito, tranne che ha subito, da parte dei medici che lo hanno curato a forza, trattamenti nocivi alla salute e non dettati dall'arte" (Stat. 296b-c. Traduzione di C. Mazzarelli).

La competenza del medico non è solo strumentale e, per questa ragione, non può essere ridotta ad un congiunto di norme. Se il medico decide di curare gli occhi attraverso l'azione di un unguento, la sua determinazione è frutto della conoscenza degli occhi, della natura della visione e della conoscenza generale di "ciò che è sano e di ciò che è malato" (Lachete 185c-d; 190; cf. Charm. 171a-b).

D'altro canto, il corpo di leggi è conditio sine qua non affinché una società possa costituirsi come tale, ma la scienza politica è nel governante, fonte della legislazione stessa (Stat. 297e). ${ }^{27}$ L'eccellenza professionale del governante sui governati è posta chiaramente in luce da Platone tanto nel Politico quanto nelle Leggi:

"Non lasciamoci convincere, cari amici, che ci sia un altro mezzo più spiccio e più facile per rivoluzionare le leggi di una città di quello messo in atto dall'autorità che ha il potere; difatti, né ora ne esiste uno, né ve ne sarà uno in futuro. E però non è questa, a nostro avviso, l'evenienza improbabile e difficile a realizzarsi; è piuttosto quest' altra, rara anche considerando un lungo lasso di tempo, ma che quando capita è causa di infiniti beni di ogni genere per la città nella quale si genera [...]. D'altra parte, questo medesimo discorso può estendersi ad ogni forma di potere. $\mathrm{E}$ difatti, quando in un uomo il sommo potere fa tutt'uno con il senno e la temperanza, ecco sorgere spontaneamente la costituzione migliore e le leggi altrettanto perfette, altrimenti nulla di ciò potrà mai avverarsi" (Leges 711c-712a).

Nel Politico gli attributi determinanti la figura e l'azione del governante emergono subito dopo il racconto del mito di Crono. Nell'era do Crono la divinità amministra in modo diretto la società. Si tratta di una società ideale in cui si vede realizzata la forma di unità perfetta. È solo dopo che la divinità ha abbandonato il mondo che gli uomini devono apprendere ad organizzarsi in modo da formare una unione a partire dalla molteplicità delle discordanze. La differenza fra il governo di Crono e il governo politico consiste nel fatto che il politico non è un dio. "Questa figura (schema), quella del pastore divino, è ancora troppo grande per essere applicata al re (he kata basilea)" (Stat. 275c). Di fatto, i governanti umani sono molto più affini, per natura ed educazione, ai propri sudditi. Il valore che godono agli occhi dei cittadini è derivato loro dal possesso di alcune doti

\footnotetext{
${ }^{27} \mathrm{Si}$ legga al proposito Gerbier 2003, in particolare 10-13 della versione on-line.
} 
Journal of Ancient Philosophy ISSN 1981-9471 - FFLCH/USP www.revistas.usp.br/filosofiaantiga
J. anc. philos. (Engl. ed.), São Paulo, v.13, n.2. p. 35-64, 2019.

DOI: http://dx.doi.org/10.11606/issn.1981-9471.v13i2p35-64

naturali, dal livello superiore di educazione cui sono soggetti, dal dominio e uso della dialektike techne, dell' empeiria, e della phronesis (Rep. 433b; 482d; 484d). La migliore costituzione rimane quella che maggiormente si approssima all'unità del governo divino (Leges 713b), ma il governante, diversamente dalla divinità, non è di natura soprannaturale e non gode della presenza di dei inferiori per gestire i raporti umani. Gli unici mezzi di cui dispone sono le norme generali e legislative. Il filosofo é differente e superiore alla massa incolta, ma pur essendo di un'altra razza, rimane, tuttavia, un membro della stessa specie.

"Prova da sua diferença é que, para que possam operar a mistura de personalidades discutida na Coda, têm, eles próprios, de ser já o arquétipo de cidadão que pretendem operar, o homem acabado. Do filósofo se pode dizer o que Jack, não por acaso i líder dos aero-náufragos de Lost, tem tatuado em caracteres chineses: "He walks among us, but he is no one of us". O político é (e nesse sentido a crítica do Estrangeiro é valida) e não é como os seus súbditos [...]. Não se separa aqui, parece-nos, o pastor divino do político humano, mas o pastor divino do também pastor humano" (Loureiro 2011, 123-124).

Il governante è legittimato a sovrintendere (epistatein) perché è dotato della scienza politica e non è obbligato ad ottenere il consenso dei sudditi, ma può anche ricorrere alla forza per imporre le proprie decisioni (Stat. 296a4-c3). E, come abbiamo indicato poco sopra, il paradigma di riferimento è, ancora una volta, la competenza medica.

Ora, al di là del grado di cognizione, altre peculiarità contrassegnano la prossimità fra medico e governante. Platone, nella Repubblica prende a prestito una convinzione già presente e giustificata nei trattati ippocratici. Socrate ammette che un medico può essere definito propriamente tale se fin dall'inizio accanto allo studio della scienza (techne) ha un contatto con i malati (Rep. 408c6-e2) $)^{28}$, perché la medicina è considerata, già da Ippocrate, una forma di "saper fare" che si prende cura dell'uomo, differente della filosofia e della religione (Mesquita 1998, 82-84). La politica, dal canto suo, non è precisamente un "saper fare" ma, come afferma Migliori, un "saper far fare" (Migliori

\footnotetext{
${ }^{28}$ Ora, è importante sottolineare che il ricorso all'osservazione non è la condizione necessaria per acquisire la scienza. Eventualmente, il rapporto è inverso: l'empeiria è necessaria per la corretta applicazione del sapere medico ai casi individuali, dal momento che le descrizioni generali non sono sufficienti per conferire al tecnico la competenza per poterle correttamente applicare. Su questo punto si veda l'articolo di Cambiano 2013, disponibile on line.
} 
Journal of Ancient Philosophy ISSN 1981-9471 - FFLCH/USP www.revistas.usp.br/filosofiaantiga
J. anc. philos. (Engl. ed.), São Paulo, v.13, n.2. p. 35-64, 2019.

DOI: http://dx.doi.org/10.11606/issn.1981-9471.v13i2p35-64

1996, 23-25). Nel Politico, infatti, lo Straniero classifica la scienza politica come una scienza gnostike epitaktike, conoscitiva direttiva, non immediatamente pratica e che, ciononostante, ha influenza diretta sulla realtà. "A ciência política é diretiva e, se ela é verdadeiramente régia, ela mesma não deve agir; não é uma ciência prática, mas deve comandar todas as ciências que têm capacidade de agir" (Dixsaut 2017, 336). La scienza politica non è kritike, solamente teorica, perché non si limita a osservare, descrivere e analizzare (krisis) una situazione (Loureiro 2011, 25-27). La politica, in quanto scienza direttiva, si riveste di un momento teorico di valutazione dei fatti, a cui si aggiunge la necessità di operare dei cambiamenti concreti nella realtà.

Come mostra il Menone 90c-92a, la validità di una tecnica si misura sulla capacità di produrre efficaci cambiamenti nella corretta direzione. Il libro X della Repubblica fa eco alle parole del Menone ricordando che ciò che accomuna la medicina e il governo dello stato è l'azione poetico/poietica che producono $(R .599 \mathrm{c}-\mathrm{d})$. Questa è la ragione per cui medicina e politica sono distinte da pittura e musica: le prime "hanno in comune con la natura la stessa azione generatrice" (Leges 889d; cf. Stat. 305d).

Nella famosa "Apologia di Protagora" (Tht. 166a-168c) l'analogon fra medico e politico si gioca, precisamente, sulla capacità di cambiamento (metabole, metableteon) che le due arti esercitano: il medico produce cambiamenti nella hexis umana attraverso le terapie (pharmakois) efficaci ad interrompere un decorso per crearne uno differente; il politico, a sua volta, produce trasformazioni nelle doxai umane attraverso l'uso prudente di discorsi (logoi) in-formativi. In queste pagine del Teeteto, hexis e doxa sono legate e coerenti l'una all'altra, in modo tale che il cambiamento di uno dei due elementi provoca inevitabilmente una rispercussione nell'altro (Marino 2018, 356-361).

\section{§ 4) Scienza e forza versus esperienza e forza}

Il rapporto che il professionista che cura adotta con il paziente discrimina, secondo Platone, i medici, nell'uso rigoroso del linguaggio, dagli assistenti dei medici (hyperetai) e lo stesso tipo di relazione può essere adoperato per discriminare il buon governo dal governo tirannico.

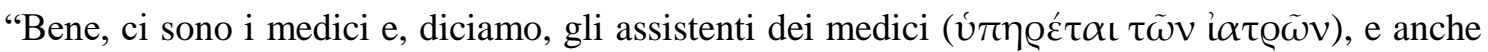
questi li chiamiamo medici. [...]. Che siano liberi o schiavi, costoro apprendono l'arte seguendo 
Journal of Ancient Philosophy ISSN 1981-9471 - FFLCH/USP www.revistas.usp.br/filosofiaantiga
J. anc. philos. (Engl. ed.), São Paulo, v.13, n.2. p. 35-64, 2019.

DOI: http://dx.doi.org/10.11606/issn.1981-9471.v13i2p35-64

gli ordini dei padroni e basandosi sull'osservazione e sull'esperienza, non secondo la natura, come invece fanno i liberi, che hanno imparato essi stessi e che insegnano ai propri figli. Puoi convenire che queste sono due tipologie di quelli che chiamiamo medici? [...]. Rifletti dunque anche sul fatto che, essendoci nella città schiavi e liberi che si ammalano, in genere sono gli schiavi a curare gli schiavi correndo a visitarli o aspettandoli negli ambulatori, e che nessuno di tali medici dà o riceve indicazione alcuna in merito alle singole malattie di questi servitori, ma dopo aver prescritto in base all'esperienza ciò che gli sembra opportuno, come se fosse perfettamente competente e con l'alterigia di un tiranno, fa un salto e se ne va da un altro schiavo malato e cosí allevia al padrone la cura dei malati.

Il medico di condizione libera, invece, generalmente cura e indaga le malattie dei liberi e studiandole dal principio secondo la natura e facendone partecipe il malato e i suoi cari impara qualcosa egli stesso dai malati e nel contempo impartisce nozioni all'infermo per quanto gli è possibile, e non dà alcuna prescrizione prima di averlo convinto: solo allora, rendendo docile l'ammalato, tramite la persuasione e un'assidua preparazione, cerca di restituirlo alla perfetta

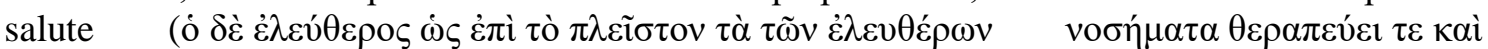

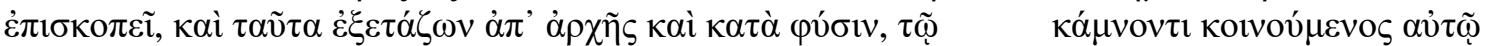

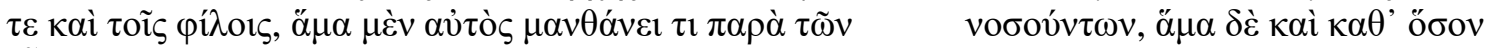

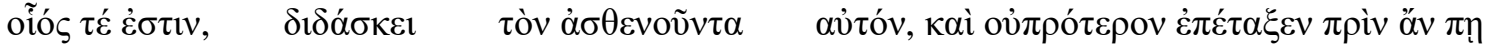

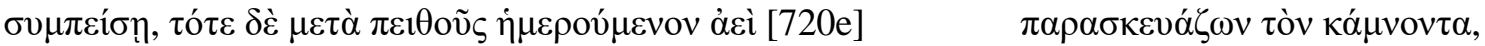

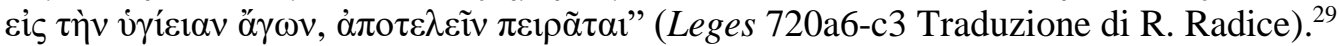

Nel passo citato, Platone distingue due tipi di medici, l'uno è il medico che, nel senso rigido del termine, cura gli ammalati perché gode della scienza, quindi della competenza, e della pratica medica che gli permette di persuadere l'individuo a rispettare le prescrizioni in vista del suo proprio bene; l'altro è una immagine distorta del professionista, in quanto si limita ad offrire una manodopera, agisce per intuizione e grazie alle numerose esperienze accumulate. Il suo modo di realizzare la professione non è guidato dalla conoscenza, ma da una opinione radicata sulla reiterata osservazione empirica.

La stessa distinzione fra l'originale e la sua immagine distorta si trova in un contesto politico nel Menone (99b-100c), nell'atto cui Socrate distingue il politico autentico, colui che possiede un sapere, quindi la scienza politica, da quanti si fanno

29 Cf. Leges 857c4-e1: "Non abbiamo dato una cattiva rappresentazione quando abbiamo assimilato tutti coloro che sono ora regolati da leggi a schiavi per così dire curati da schiavi. Infatti, occorre sapere bene tale cosa, cioè che se mai un medico, tra coloro che praticano l'arte medica in base a loro esperienza ma senza teoria, incontrasse un medico libero mentre sta dialogando con un malato libero, valendosi di discorsi vicini a quelli propri dalla filosofia e occupandosi della malattia fin dal suo inizio, risalendo alla natura generale dei corpi, subito scoppierebbe in una gran risata e non farebbe discorsi diversi da quelli che su tali questioni sono sempre sulla bocca della maggior parte dei cosiddetti medici. Infatti direbbe: Sciocco non curi il malato, ma per così dire lo educhi (paideueis), come se avesse bisogno di diventare un medico, non di diventare sano". 
Journal of Ancient Philosophy ISSN 1981-9471 - FFLCH/USP www.revistas.usp.br/filosofiaantiga
J. anc. philos. (Engl. ed.), São Paulo, v.13, n.2. p. 35-64, 2019.

DOI: http://dx.doi.org/10.11606/issn.1981-9471.v13i2p35-64

chiamare comunemente politici, i quali agiscono in virtù di una retta opinione digiuna di riflessione (phronein). ${ }^{30}$

"Potremmo giustamente chiamare divini coloro che poco fa dicevamo vaticinatori e vati tutti quanti i poeti; e non meno di costoro potremmo dire che sono divini i politici, e che sono invasati, quando sono ispirati e posseduti dal dio, allorché hanno successo nel dire molte grandi cose, senza sapere nulla di ciò che dicono (meden eidotes on legousin)" (Men. 99b. Traduzione di U. Bultrignini).

Il medico degli schiavi non conosce la natura umana, né l'origine delle patologie e tratta tutti i pazienti in modo impersonale. L'empeiria di cui si serve nasce sulla base del ricordo di una serie di casi simili senza la necessità di risalire ai fattori causali (Leges IV 720b2-5; c3-6). La funzione terapeutica della psicagogia rappresenta un fattore irrilevante in queste condizioni. L' uso della parola è lo strumento di forza di cui si serve. Si tratta, in tal caso, di un logos retorico non dissimile all'adulazione sofistica. I medici degli schiavi sono una specie di infermieri (hyperetai) che per pratica (empeiria) impongono prescrizioni al paziente, sempre le stesse in circostanze simili, incapaci come sono di fornire una giustificazione (aitian ekastou). Tuttavia, se sottoposti ad una osservazione attenta, tanto il medico propriamente detto, quanto il medico qualitativamente inferiore, si servono dello stesso mezzo per ottenere l'obbedienza del malato: la forza della persuasione contenuta nel discorso. Perciò, al fine di distinguere l'originale dalla sua imitazione, è necessario addentrare un livello più profondo di analisi del discorso persuasivo.

L'efficacia del discorso è depositata nella forza che esso esercita, come è evidenziato nel Gorgia (514d). Nel tentativo socratico di definire la retorica, le tecniche sono classificate in funzione della loro relazione con la parola e il ruolo ad essa attribuito. All'inizio del dialogo, Gorgia aveva difeso la superiorità del retore sul medico quando si trattasse di convincere il popolo ad eleggere il medico pubblico. Se l'elezione del medico fosse stabilita dai voti dei cittadini all'interno di una assemblea pubblica, certamente l'incanto della parola retorica avrebbe la meglio sulla competenza laconica del professionista nella salute.

\footnotetext{
${ }^{30}$ Per maggiori dettagli sulla distinzione fra le due figure in ambiete politico si veda Lisi 2011, 204.
} 
Journal of Ancient Philosophy ISSN 1981-9471 - FFLCH/USP www.revistas.usp.br/filosofiaantiga
J. anc. philos. (Engl. ed.), São Paulo, v.13, n.2. p. 35-64, 2019.

DOI: http://dx.doi.org/10.11606/issn.1981-9471.v13i2p35-64

Da queste considerazioni è possibile guadagnare elementi sintomatici per la presente indagine: la prossimità fra medico e sofista; la facilità di confondere il politico, il sofista, il medico e l'infermiere; e l'importanza della retorica in generale, il cui valore etico dipende dai fini per i quali è utilizzata.

La distinzione promossa da Platone fra l'uomo dotato di scienza, che attinge l'obiettivo anche grazie all'uso consapevole della parola persuasiva, e l'uomo dotato di forza, che realizza il suo compito attraverso l'imposizione, è sottile, dal momento che l'obbedienza attinta grazie alla persuasione, resta, comunque, un atto di forza. E anche se fosse in vista del bene del paziente, questo atto rimarrebbe pur sempre un atto di violenza. Non è possibile condizionare un comportamento senza automaticamente trattare il soggetto come un inferiore.

"A questão é tanto mais incómoda quanto mais se partilha a ideia de que a razão é uma, como os antigos criam [...], ou seja, que duas pessoas racionais chegam necessariamente à mesma conclusão pelo uso recto da razão" (Loureiro 2011, 60 nota 65).

In queste condizioni, il ricorso alla forza persuasiva equivale al riconoscimento dell'inferiorità dell'interlocutore.

L'arte del governo non è mai esente dall'uso della forza, come afferma l'Ateniese nelle Leggi (711c-712a). Le relazioni di comunicazione e persuasione, da un lato, e di imposizione, dall'altro, non sono tanto distanti come sembra a prima vista. Prova di ciò è il fatto che sono rese in greco dall'uso di un unico verbo la cui radice é peith- (peitho e peithomai). Come nota Sabrina Grimaudo,

"In questa dinamica un ruolo essenziale è svolto dalla persuasione (peitho) che, peraltro, com'è noto, presenta nella cultura greca uno statuto non univoco e anzi talora ambivalente. Basti considerare il fatto che le prime attestazioni del verbo peithomai (al medio-passivo), nei poemi omerici, rimandano al significato di 'obbedire' laddove l'attivo peithei 'persuadere' 'convincere' sembra essersi sviluppato solo a partire dal medio: come dire che tra persuasione e obbedienza esiste, fin dalle origini della cultura greca, un rapporto complesso e non unidirezonale" (Grimaudo $2014,35){ }^{31}$

\footnotetext{
${ }^{31}$ Il rapporto di comunicazione fra medico e malato caratterizza diversi trattati ippocratici, ma non ricopre la totalità di essi. Secondo l'autore di Medicina Antica II 3 il prognostico medico deve collimare con la descrizione che il malato fornisce dei suoi propri disturbi. Non solo, il medico deve utilizzare un linguaggio semplice in modo che le sue parole siano intese (gnosta) da un profano (demotisin). Si veda anche Giuramento del medico. Una relazione differente è auspicata, invece, nel trattato De Arte, e si tratta di una relazione di imposizione e di comando. Al paziente non è attribuita nessuna fiducia da parte del medico, il quale pertanto resta sordo alle descrizioni
} 
Journal of Ancient Philosophy ISSN 1981-9471 - FFLCH/USP www.revistas.usp.br/filosofiaantiga
J. anc. philos. (Engl. ed.), São Paulo, v.13, n.2. p. 35-64, 2019.

DOI: http://dx.doi.org/10.11606/issn.1981-9471.v13i2p35-64

Malgrado questa evidenza, rimane una nota distintiva nei due tipi di professionista, legata all'uso peculiare che viene fatto del logos.

"A diferença entre governo violento ou aceite parece-nos sobretudo uma extensão do anterior corte entre o pastor humano e divino: só este último, de fato, reina pela persuasão. O príncipe, como o filósofo, ocupa entre este [o deus] e o tirano (que governa só pela força), um terceiro lugar, de cruzamento entre ambos: ele impõe-se pela persuasão e pela violência ao rebanho [...]. Os governados podem ser persuadidos da bondade do que lhe é imposto, submetendo-se, mas têm de ser persuadidos primeiro. O Estado tem de dar razões de suas ações: as leis têm de ter um prêambulo. Por si, ninguém estará disposto a aceitar uma autoridade externa" (Loureiro 2011, 133-134).

La vera distinzione tra infermiere e medico e tra tiranno e re consiste nel fatto che il secondo usa la forza in unione con la scienza, l'intelligenza e il rispetto di cui il primo è sprovvisto.

\section{§ 5) Peculiarità nell'uso del discorso retorico}

L'uso di termini propriamente civici e politici per mostrare i due differenti atteggiamenti dei medici, lascia aperta l'ipotesi che il filosofo greco non volesse ridurre l'analogon fra medicina e arte di governo ad una semplice comparazione. Come fa notare Frédéric Worms nella conferenza "Que nous enseigne Platon pour la médicine aujourd'hui", ridurre l'esempio ad una relazione estrinseca impedisce di attingere la profondità del messaggio platonico che, secondo l'autore, ha come obiettivo di evidenziare come il fatto di modulare l'uso del logos sull'interlocutore è necessario per riconoscere e preservare la libertà dell'individuo.

Il medico degli schiavi si serve di un discorso e di una retorica incapace di giustificarsi. Perciò, si tratta di un logos teatrale più che di un logon didonai (Cambiano 2013, 3 della versione on-line). Del resto, con la folla incolta non resta che azionare un artificio sofistico.

fornite dai malati. In questo caso, il medico ordina, e al paziente non resta che obbedire e se la cura non attinge l'esito sperato, la colpa deve essere attribuita all'incompetenza del malato o alla pigrizia nel seguire gli ordini del superiore. Interessante è la formulazione delle Epidemie I 2, 5, in cui la relazione non è biunivoca ma tradica: il medico si fa alleato del paziente nella lotta contro la malattia. Sulla differenza nelle relazioni fra medico e malato si veda Grimaudo 2014, 36 e nota 7. 
Journal of Ancient Philosophy ISSN 1981-9471 - FFLCH/USP www.revistas.usp.br/filosofiaantiga
J. anc. philos. (Engl. ed.), São Paulo, v.13, n.2. p. 35-64, 2019.

DOI: http://dx.doi.org/10.11606/issn.1981-9471.v13i2p35-64

Contrariamente al medico che lavora tais empeiriais aneu logou (Leges 857c7e1), il medico degli uomini liberi fa un uso criterioso della parola, entra in simpatia (koinoumenos, Leges 720c1) con il malato e i suoi familiari, stabilisce la comunicazione reciproca e disponibilizza le proprie conoscenze.

"Par conséquent, dans la mesure où il en est capable, le médicin véritable enseigne (didaskei) au malade lui-même (mais apprend aussi, manthanei, du malade) et ne lui administre aucun remède avant de l'avoir persuadé et ainsi prédispose à accepter sa thérapie" (Cambiano 2013, 4 della versione on-line).

Il suo discorso assomiglia a ciò che nel Gorgia Platone qualifica come un tipo di retorica peithodidaskalike (Gorg. 452e, 453d-455a, 456b1-6). Questo aggettivo, che rivela il valore di un certo tipo e di un certo uso della retorica, è rievocato nel Fedro, alle pagine $270 \mathrm{a}-274 \mathrm{a}$, in cui Socrate approssima la techne iatrike alla techne retorike. ${ }^{32}$ Trattasi anche in questo passo di una retorica differente da quella che nel Gorgia (463a466a) è legata alla culinaria (Ttrabattoni 1994, 102-106). Infatti, è in virtù del fatto che il medico conosce le proprietà degli alimenti e la natura umana, che egli può usare il discorso persuasivo al lato e in funzione complementare alla terapia.

Il fatto che il medico degli uomini liberi conosca le cause della malattia e sia in grado di spiegarle al paziente (“... indaga le malattie dei liberi e stadiandole dal principio secondo la natura e facendone partecipe il malato, Leges, 720c3-5), agendo così in modo persuasivo, fa sì che quest'ultimo non si senta umiliato nel suo valore di uomo libero. In queste condizioni è rispettata la possibilità di scelta del malato, il quale può ricorrere o meno al trattamento del medico, può acconsentire o rifiutare la cura impartitagli (Cambiano 2013, 5 della versione on-line). L'idea è anticipata nella Repubblica, nel passo in cui Platone stabilisce che spetta al malato di rivolgersi al medico e non viceversa $(R$. 498a-e). "Non sta a colui che comanda di pregare colui che è tenuto ad obbedirgli, così come non spetta al medico pregare il malato di sottoporsi alle sua cure" (Lombard 2016, $15)$.

Il medico degli uomini liberi si prende cura di uomini "liberi”, perché non ignora la dimensione umana e libera del paziente. Il processo ermeneutico, formato di comprensione e spiegazione, coinvolge entrambe le parti in causa in quanto tanto il

\footnotetext{
${ }^{32}$ La relazione suddetta è problematizzata in Vegetti 1995.
} 
Journal of Ancient Philosophy ISSN 1981-9471 - FFLCH/USP www.revistas.usp.br/filosofiaantiga
J. anc. philos. (Engl. ed.), São Paulo, v.13, n.2. p. 35-64, 2019.

DOI: http://dx.doi.org/10.11606/issn.1981-9471.v13i2p35-64

medico come il paziente si trovano nella condizione di spiegare e capire. Un medico libero "conversa con il malato da uomo libero e parla con termini che si approssimano a quelli della filosofia" al punto da essere accusato di voler fare del paziente "non un uomo sano, ma un medico" (Leges 857c-e). Si evita così di trattare l'interlocutore come uno schiavo, privandolo di esercitare la propria libertà. Nella misura in cui il medico accoglie la comprensione del paziente, si approssima all'individuo e, a sua volta, ricorre a varie forme di spiegazione e comprensione della situazione (Caprara 1999, 649). Il medico degli uomini liberi si prende cura ad un tempo delle tre dimensioni che costituiscono l'individuo: la dimensione fisica, psichica e politica.

La relazione medico - paziente è posta da Platone in funzione di chiarire i fondamenti su cui edificare la citta giusta. L'analogon è utilizzato al fine di evidenziare le conseguenze prodotte da due modi di reggere lo stato. ${ }^{33}$ Ciò che distingue il politico dal tiranno non è l'esercizio dell'ufficio pubblico, bensì la capacità e il dovere di rendere ragione delle decisioni assunte in relazione alla comunità. È in virtù del fatto che il politico possiede la scienza del governo e conosce la natura dei logoi che egli sa a chi può indirizzare le proprie parole (Marino 2018, 353). In questo modo, il cittadino non si sente costretto ad obbedire ad un imperativo etico, ma ossequia un dovere che egli proprio si dà. Tuttavia, è bene non azzerare la supremazia del medico e del governante sui subordinati. Infatti, come è detto nel Politico, se gli uomini fossero in grado di discutere di ciò che è bene per loro, il corpo legislativo non sarebbe necessario (cf. Stat. 296a-300c, Lombard 2016, 51). Non è opportuno ignorare la differenza che esiste fra curare, istruire ed educare gli uomini. Per questo, il tratto che valorizza la legislazione delle Leggi è il preambolo (prooímion), ossia la giustificazione del provvedimento adottato, e non il contenuto delle singole leggi. Il proclama legislativo assume una funzione terapeutica e psicagogica, nel momento in cui indica ai cittadini i buoni e i cattivi "regimi", avvalendosi di una retorica filosofica (cf. Leges 711c). Se l'obbiettivo del legislatore è mantenere la salute della società, è necessario che i cittadini vengano persuasi relativamente alla bontà

\footnotetext{
${ }^{33} \mathrm{Nel}$ caso della relazione medicina - política, Platone si serve frequentemente dello strumento dell'analogia. Ben nota è la descrizione dei processi patologici dell'individuo applicati analogicamente ai processi che ricorrono nella città e nell'anima (Marino 2018, in particolare 349-350; cf. Lloyd 1992).
} 
Journal of Ancient Philosophy ISSN 1981-9471 - FFLCH/USP www.revistas.usp.br/filosofiaantiga
J. anc. philos. (Engl. ed.), São Paulo, v.13, n.2. p. 35-64, 2019.

DOI: http://dx.doi.org/10.11606/issn.1981-9471.v13i2p35-64

di un provvedimento assunto non solo in vista dell'esistenza pacifica della città, ma per il bene dell'individuo.

"Com esse fim, Platão concebe um instrumento legislativo inédito, o prêambulo: o legislador deverá fazer certas leis serem precedidas de um discurso preliminar, dirigido ao cidadão e destinado a persuadi-lo, pela exortação e pela ameaça, a censurar o comportamento contrário à lei que se segue ao prêambulo e louvar o comportamento que ela autoriza. Cabe ao "prêambulo" favorecer essa univocidade no elogio e na censura que o início do dialogo afirmava ser o objetivo do legislador" (Brisson e Pradeau 2012, 86).

Il preambolo è una sorta di "preludio" musicale, per usare le parole di Brisson e Pradeau, il cui obiettivo è di legittimare il provvedimento adottato. L'espressione utilizzata dagli studiosi francesi è particolarmente apppropriata, visto che Platone sembra avere in mente dei prologhi cantati. Il filosofo usa, infatti, il termine epodai per indicare i preamboli alle leggi che i cittadini dovranno intonare e incastonare nelle loro anime (Leges 793a9-d5). ${ }^{34}$ Lo stesso termine ricorre nel Carmide per indicare la terapia necessaria a trattare la follia. Questa malattia dell'anima viene curata quando al movimento fisico si affiancano le epoidai, le odi, ossia, i discorsi belli (kaloi logoi), in quanto esse favoriscono il ripristino della sophrosyne (Charm. 155e-157b).

Il testo della legge, privato dell'epode del preambolo, non è in grado di giustificare il proprio contenuto, dal momento che esso si presenta attraverso asserzioni che obbligano, prescrivono, costringono e minacciano. In altre parole, la legge agisce con la forza, ma la forza senza persuasione provoca la ribellione (Casertano 2011, 80-81).

Il testo propedeutico ha principalmente due funzioni: la prima è preventiva. Esattamente come il compito della medicina è da un lato profilattico e dall'altro terapeutico, allo stesso modo il preambolo non si dirige immediatamente al colpevole, ma al cittadino in generale, al fine di prevenire un comportamento nocivo, mentre la legge si dirige direttamente al colpevole per indicargli la pena. La seconda funzione del preambolo è di sostituire all'uso della forza la leggerezza della parola e, forse, della musica, trattandosi di epodai. Del resto, ciò che il preambolo si propone è precisamente "obter, qualquer que seja a lei que ele preceda, a adesão dos cidadãos. [...] Como o Ateniense explica em VIII, 840b-e, o prêambulo é uma espécie de encantamento coletivo que se

\footnotetext{
${ }^{34} \mathrm{Si}$ veda Senofonte, Memorabili IV, 4, 14. Su questo punto, particolarmente interessanti sono le riflessioni di Dixsaut 2017, 342-346 e 346 nota 16.
} 
Journal of Ancient Philosophy ISSN 1981-9471 - FFLCH/USP www.revistas.usp.br/filosofiaantiga
J. anc. philos. (Engl. ed.), São Paulo, v.13, n.2. p. 35-64, 2019.

DOI: http://dx.doi.org/10.11606/issn.1981-9471.v13i2p35-64

propõe a inspirar um respeito coletivo suficientemente eficaz” (Brisson e Pradeau, 2012, 91).

Ciò che corona la legislazione presentata nell'ultima opera platonica è il fatto che tutto il corpo legislativo, preambolo e legge, deve agire primordialmente e direttamente sull'anima. Del resto, la legislazione condivide con l'anima la medesima natura: entrambe sono movimento detto e ascoltato. ${ }^{35}$

È interessante, a questo proposito, evidenziare il fatto che tanto che i proemi legislativi come le leggi hanno una relazione con la musica. Il prooimon in origine evoca il canto. Infatti, con il termine oime i Greci indicavano l'inno iniziale che veniva cantato in apertura ad un poema epico (Ferrari 2016, 203-205 e 207-209). ${ }^{36}$ Il termine legge, nomos, a sua volta, non è estraneo all'universo musicale. Assume, infatti, fra i molteplici significati attribuitigli nelle Leggi, anche quello di canto:

"Risulti stabilita dunque, diciamo, questa stranezza, che i canti sono per noi leggi, e infatti gli antichi chiamarono in tal modo il canto accompagnato dalla cetra, cosicché forse neppure essi sarebbero del tutto lontani da ciò che ora diciamo" (Leges 799e-800a. Traduzione di R. Radice).

Nonostrante, il carattere musicale delle leggi, esse rimangono, però, norme gestite dalla parte razionale dell'anima (Stat. I 644c5-10). ${ }^{37}$ Perciò è necessario che esse siano introdotte dal preambolo persuasivo, che toglie all'imposizione della legge il carattere di un imperativo categorico.

\section{Conclusione}

\footnotetext{
${ }^{35} \mathrm{Si}$ legge nel frammento di Ateneo, XIV 628c (=37B6 DK): "Con ragione Damone ateniense diceva che i canti e le danze necessariamente si generano da un certo movimento dell'anima; e quei canti che sono liberi e belli creano tali le anime; quelli contrari, le contrarie". Per approfondimenti si veda FerrariI 2016, 177.

${ }^{36}$ Sull'importanza della musica in vista dell'edducazione ética e politica e come elemento di armonia fra corpo e anima, rimandiamo all'eccellente lavoro di Ferrari 2016, le cui profonte analisi ci hanno offerto numerosi spunti di riflessione i cui riflessi si trovano nel presente testo. Siamo inoltre grati all'autore per l'eccellente bibliografia indicata e da noi utilizzata per approfondire i nostri studi.

${ }^{37}$ L. I 644c8-10: "Al di sopra di tutti questi stati d'animo si ha uma sorta di calcolo su quale di essi è migliore e su quale è peggiore, e questo calcolo, una volta affermatosi come credenza collettiva di una città, prende il nome di legge".
} 
Journal of Ancient Philosophy ISSN 1981-9471 - FFLCH/USP www.revistas.usp.br/filosofiaantiga
J. anc. philos. (Engl. ed.), São Paulo, v.13, n.2. p. 35-64, 2019.

DOI: http://dx.doi.org/10.11606/issn.1981-9471.v13i2p35-64

Nel corso dei dialoghi platonici la medicina raramente manca dalla lista delle arti ed è oggetto di analisi puntuali, al punto da infastidire non pochi interlocutori di Socrate, come è il caso di Callia nel Gorgia: "Per gli dei! Continui a parlare proprio di calzolai, di cardatori, di cuochi e di medici, come se il nostro ragionamento riguardasse costoro" (490e-491a).

Nonostante il fatto che la relazione fra medicina e politica non sia una invenzione di Platone e sia già stata oggetto di studio da parte di molti esegeti, in questo testo ci siamo proposti di evidenziare fino a che punto l'attenzione di Platone per la medicina abbia potuto influenzare il suo modo di parlare e di pensare anche in ambiti apparentemente estranei ad essa. In vista di ciò, abbiamo ripreso alcuni aspetti da noi ritenuti meno esplorati negli studi anteriori, partendo dall'analisi di alcuni passi tratti dal Timeo, dal Politico e dalle Leggi.

L'obbiettivo dello studio è stato di mostrare che l'analogon fra medicina e politica negli scritti platonici è sostanziale, levando in considerazione le dimensioni terapeutica e civica che entrambe le scienza condividono. Nel corso del testo abbiamo dapprima presentato le due figure e abbiamo esposto le difficoltà di definire chi meriti il titolo di medico vero e di politico autentico, mettendo in luce gli attributi che distinguono il professionista dalla sua immagine fallace. Abbiamo, quindi, analizzato la costituzione peculiare dell'oggetto di studio delle due scienze, ossia le dimensioni fisico - psichica e politica costitutive dell'uomo; e i mezzi di cui le due arti si servono per plasmare un certo tipo di uomo, dopo aver fornito alcune informazioni sulla struttura epistemologica delle due scienze.

Barbara Botter

Universidade Federal do Espírito Santo

\section{Bibliografia}

Platone, Timeu/Crítias. Trad. e com. L. Brisson. Paris: Flammarion, 1992, 2001³ . 
Journal of Ancient Philosophy ISSN 1981-9471 - FFLCH/USP www.revistas.usp.br/filosofiaantiga
J. anc. philos. (Engl. ed.), São Paulo, v.13, n.2. p. 35-64, 2019.

DOI: http://dx.doi.org/10.11606/issn.1981-9471.v13i2p35-64

Platone, Timeo. Trad. F. Fronterotta. Milano: BUR 2015. https://doi.org/10.1515/ 9783110240801.53

Platão, O Político. Trad. Carmen Isabel Leal Soares. Lisboa: Temas e Debates. Circulo Leitores 2008. https://doi.org/10.14195/2183-1718_60_40

Platone, As Leis. Trad. e com. E. Bini. Bauru, São Paulo: EDIPRO 1999.

Reale G. (a cura di), Platone. Tutti gli scritti. Milano: Rusconi 1991.

Annas J., An Introduction to Plato's Republic. Oxford: Oxford University Press 1981. https://doi.org/10.2307/2184431

Biral A. La felicità. Lezioni su Platone e Nietzsche. Padova: Il Prato 2005.

Bourgey L. Observation et expérience chez les médicins de la Collection hippocratique, Trisième partie, Cap. I, La médicine rationnelle. Paris: Vrin 1953.

Brisson L., Pradeau J.F., (eds.), As Leis de Platão. São Paulo: Loyola 2012.

Brisson, L, Le Même et l'Autre dans la structure ontologique du Timée de Platon. SanktAugustin: Academia Verlag 1998, $2015^{4}$.

Brisson, L. Platon. Le Politique. Introd., Trad., et notes. Paris: Flammarion 2003. https://doi.org/10.1522/cla.pla.pol

Brisson, L. Haw and why do the building blocks of the universe change constantly in Plato's Timaeus. In Nataçli C., e Maso ST. (org.), Plato Physicus. Amsterdam: Hakkert 2003, 198-207.

Cambiano G., Platon et les rapports entre théorie et praxis dans la médicine hippocratique. Études platoniciennes. 10, 2013. Mise en ligne em mars 2014. Consulté le 6 de avril 2018.

Brisson, L. Platone e le tecniche. Roma: Editora Laterza, 1991.

Carone, G. A cosmologia de Platão e suas dimensões éticas. São Paulo: Loyola 2008.

Caprara A. A relação médico - paciente. Para uma humanização da prática médica. Cadernos de Saúde Pública. 15 (3), 1999, 647-654.

Capriglione J. La diaita secondo Galeno. Cuadernos de Filologia Clásica. 10, 2000, 155-172.

Casertano, G. Introdução à República de Platão. São Paulo: Paulus 2011.

Casertano, G. Il nome della cosa. Linguaggio e realtà negli ultimi dialoghi di Platone. Napoli: Loffredo Editore 1996.

Casertano, G. L'eterna malattia del discorso. Quattro studi su Platone. Napoli: Liguori Editore 1991.

Castoriadis C., On Plato's Stateman. Stanford: Stanford University Press 2002.

Del Forno D., La struttura numerica dell'anima del mondo (Timeo 35 B 4-36 B 6), Elenchos XXVI 2005, 3-32.

Desclos, M-L. Représentation médicale du public et publicité de la médicine. In MACÉ, A. (org.), Le savoir Public: la vocation politique di savoir en Gréce ancienne. Paris: Presses Universitaires de France-Comté, 2013, 201-234.

Dixsaut M. (ed.), Études sur la Republique de Platon. 2 voll. Paris: Vrin 2005.

Dixsaut M. "Natura e ruolo dell'anima nella sensazione", in G. CASERTANO (a cura di), $I l$ Teeteto di Platone: struttura e problematiche, Loffredo, Napoli, 2002, 39-62.

Dixsaut M. Platão e a questão da alma. São Paulo: Paulus 2017. 
Journal of Ancient Philosophy ISSN 1981-9471 - FFLCH/USP www.revistas.usp.br/filosofiaantiga
J. anc. philos. (Engl. ed.), São Paulo, v.13, n.2. p. 35-64, 2019.

DOI: http://dx.doi.org/10.11606/issn.1981-9471.v13i2p35-64

Dixsaut M. Platon - Nietzsche. L'autre manière de philosopher. Paris: Fayard 2015.

Dulce Reis, M., "A relação entre a teoria da tripartição da alma e a teoria ético-política platônica". In CAMPOLINA DINIZ PEIXOTO M., (org.), A saúde dos antigos. São Paulo: Loyola 2009, 107-123.

Dulce Reis M., Virtude e Vício. Tripartição e Unidade da Alma no Timeu e nas Leis de Platão. Rio de Janeiro: 7Letras FAPEMIG 2010.

Ejik van der, $\mathrm{Ph}$., "Os conceitos de saúde mental na medicina e na filosofia gregas dos séculos $\mathrm{V}$ e IV a.C.”. In Campolina Diniz Peixoto M., (org.), A saúde dos antigos. São Paulo: Loyola 2009, 11-23.

Esposito R. Bios, Biopolitica e Filosofia. Lisboa: Edições 70 [2004] 2010.

Federspil G. la dieta come atto medico. - Lettura magistrale dal VI Congresso

Nazionale ANSISA - Roma 24 - 25 marzo 2000.

Ferrari F. L'antropologia di Platone: il problema della persuasione e le sue ricadute sul rapporto anima-corpo.Tesi di Dottorato, Coordinatore del Dottorato Prof. Carlo Natali , Venezia 2016.

Garcia Gual C. Dieta hipocrática y prescripciones alimenticias de los pitagoricos. In Perez Jimenez A. e Cruz Andreotti G., (eds), Dieta mediterranea. Comida y hábitos alimentícios en las culturas mediterráneas. Madrid: Ediciones Clásicas 2000, 43-67. https://doi.org/10.24277/ classica.v20i2.146

Garcia Soler M. J. "Nourriture et santé dans la medicine grecque Ancienne". No original, "la alimentación y la salud en la Grecia antigua. Vitarte. Revista cuadrimestral de humanidades. 34, 2004, 5-20. https://addi.ehu.es/.../GARCÍA\%20SOLER\%2C\%20M \%20 Acessado em 08.08.2018.

Gerbier L, La politique et la médicine : una figure platonicienne et sa relecture averroïste. Asterion. 1, 2003. Mise en ligne em avril 2005. Consulté 6 de avril 2018. https://doi.org/10.4000/asterion.13

Gomes L. B. Bolze S. D. Azeredo, Bueno R. K., Crealdi M. A., As Origens do Pensamento sistêmico: Das Partes para o Todo. In Pensando Famílias, 18(2), 2014, 3-16.

Grimaudo S., Obbedienza e persuasione. Due modelli della relazione medico - paziente nella Grecia Antica. Hormos. Ricerche di Storia antica. n. 6 2014, 34-47.

Joli R., Recherches sur le traité pseudo-hippocratique du Régime. Genève, Bibliothèque de la Faculté de Philosophie et Lettres de l'Université de Liège. Fasc. CLVI 1960.

Joubaud C. Platon et La médicine. Le corps affaibli et l'âme attristée. Paris: L'Hermattan 1999.

Jouanna J. Politique et médécine. In Hippocratica. Paris, CNRS 1980, 299-319.

Laks, A. Introdução à filosofia Pré-socrática. São Paulo: Paulus 2013. https://doi.org/10.11606/issn. 1981-9471.v10i1p100-110

Lane M. Method and Politics in Plato's Stateman. Cambridge: CUP 1998.

Lisi F. A política platônica: o governo da cidade. In Brisson L., Fronterotta F. (Org.). Platão Leituras. São Paulo: Paulus 2011, 203-219.

Lisi F. Einheit und Vielheit des Platonischen Nomosbegriffes. Beiträge zur Klassischen Philologie 167 1985, 239-243.

Lombard J. Platone e la medicina. Forlì: Victrix 1999, 2016. 
Journal of Ancient Philosophy ISSN 1981-9471 - FFLCH/USP www.revistas.usp.br/filosofiaantiga
J. anc. philos. (Engl. ed.), São Paulo, v.13, n.2. p. 35-64, 2019.

DOI: http://dx.doi.org/10.11606/issn.1981-9471.v13i2p35-64

Lopez F. Il pensiero olistico di Ippocrate. Percorsi di ragionamento e testimonianze. San Giovanni in Fiore (Cs): Pubblisfera Edizioni, 2004.

Loureiro R. P. G. João Diogo, Comentário Político - Filosófico ao político de Platão. Dissertação de Mestrado em Estudos Clássicos apresentada à Faculdade de Letras da Universidade de Coimbra 2011.

Lloyd G.E.R. Polarità e Analogia. Napoli: Loffredo 1992.

Marino S. Medicina. In CCornelli G. e Lopes F. (org.), Platão. São Paulo: Paulus 2018, 349-361. https://doi.org/10.14195/978-989-26-1596-7

Matsui S. Medicina e política: A polêmica de Platão com a medicina na República. Dissertação de Mestrado defendida na Universidade de Brasilia. Orientador, Prof. Dr. G. Cornelli, 2015.

Mesquita A. P., Entre filosofia e medicina: a antropologia hipocrática. Philosophica 11 1998, 6385.

Migliori M. "Il problema della generazione nel Timeo". In Natali C., Maso St. (orgs.), Plato Physicus. Amsterdam: Hakkert 2003, 97-120. https://doi.org/10.1163/24680974-90000454

Migliori M., Arte Politica e Metrica Assiologica. Commentario Storico-Filosofico al «Politico» di Platone. Milano: Vita e Pensiero 1996.

Morais K. L. F., A Unidade corpo-alma na fisiologia-ética do Timeu de Platão. Tese (Mestrado). Universidade Federal de Minas Gerais 2009.

Morais K. L. F. (2009a), “A relação alma-corpo no Timeu de Platão”. In Campolina Diniz Peixoto M. (org.), A saúde dos antigos. São Paulo: Loyola 2009, 123-135.

Palumbo L. Verba Manent. Napoli: Loffredo 2016.

Robinson T.M. A psicologia de Platão. São Paulo: Loyola 2007. https://doi.org/10.14195/978989-26-1596-7_15

Robinson T.M., Psicologia. In Cornelli G. e Lopes R. (org.), Platão. São Paulo: Paulus 2018, $295-$ 319. https://doi.org/10.14195/978-989-26-1596-7

Rosen S., Plato Stateman. The web of Politics. New Haven - London: Yale University Press 2003. https://doi.org/10.2307/2960172

Rosen S. Plato's Republic. A study. New Haven - London: Yale University Press 2005.

Schuhl P.M. Platon et la médicine. Revue de Études Grecques. 73, 1960, 73-79.

Soares C. Mesas terapêuticas e mesas saudáveis: Platão em diálogo com a literatura médica e gastronómica. Archai, n. 23, May-Aug., 2018, 229-264. https://doi.org/10.14195/1984249x_23_8

Soares Leal C. I., Platão. O Politico. Introdução, Tradução e Notas. Lisboa: Circulo de Escritores/Temas e Debates 2008. https://doi.org/10.14195/2183-1718_60_40_

Soares Leal C. I Crises Politicas. Reflexões de Ontem e de Hoje. In Curado A. L., \& Pereira V. S. (eds.), A antiguidade Clássica e Nós Herança e Identidade Cultural. Braga: Universidade do Minho, Centro de Estudos Humanísticos 2006, 73-78.

Soares C., Arquéstratos. Iguarias do mundo grego. Guia gastronimico do Mediterraneo Antigo. Coimbra: Imprensa da Universidade de Coimbra 2016. https://doi.org/10.14195/978-989-26$1245-4$

Thivel A. L'évolution du sens de diaita. In López Férez A. (ed.), La lengua científica griega: orígenes, desarrollo e influencia en las lenguas modernas europeas. Vol. I. Madrif: Edicíones Clásicas 2000, 25-37. 
Journal of Ancient Philosophy ISSN 1981-9471 - FFLCH/USP www.revistas.usp.br/filosofiaantiga
J. anc. philos. (Engl. ed.), São Paulo, v.13, n.2. p. 35-64, 2019.

DOI: http://dx.doi.org/10.11606/issn.1981-9471.v13i2p35-64

Trabattoni F. Scrivere nell'anima. Verità, dialettica e persuasione in Platone, La Nuova Italia, Firenze, 1994.

Volpe Cacciatore P. El régimen según Galeno. In Perez Jimenez A. e Cruz Andreaotti G., (eds), Dieta mediterranea. Comida y hábitos alimentícios en las culturas mediterráneas. Madrid: Ediciones Clásicas 2000, 91-101.

Worms Fr. Que nous enseigne Platon pour la médicine aujourd'hui. conferenza in https://www.sam-network.org/video/cours-du-16-fevrier-2016?curation=0)

Vegetti M., La medicina in Platone. Venezia: Il Cardo 1995.

Vegetti M. Fra Platone e Galeno: curare il corpo attraverso l'anima, o l'anima attraverso il corpo? Atque. Materiali fra filosofia e psicoterapia. 2015, 75-87.

Vegetti M. Um paradigma do céu: Platão político de Aristóteles ao século XX. São Paulo: Annablume, 2010. https://doi.org/10.14195/978-989-26-0946-1 